\title{
PCAP is the major known prostate cancer predisposing locus in families from south and west Europe
}

\author{
Géraldine Cancel-Tassin ${ }^{* 1,4}$, Alain Latil ${ }^{1,4}$, Antoine Valéri ${ }^{1,2}$, Philippe Mangin ${ }^{3}$, \\ Georges Fournier $^{2}$, Philippe Berthon ${ }^{1,4}$ and Olivier Cussenot ${ }^{1,4,5,6}$
}

\author{
${ }^{1}$ Centre de Recherche pour les Pathologies Prostatiques (CeRePP. UA3104 Paris VII), genopole ${ }^{\circledR}$, Evry, France; \\ ${ }^{2}$ Service d'Urologie, CHU de la Cavale Blanche, Brest, France; ${ }^{3}$ Service d'Urologie, CHU de Nancy-Brabois, \\ Vandoeuvre les Nancy, France; ${ }^{4}$ UroGène, genopole ${ }^{\circledR}$, Evry, France; ${ }^{5}$ Institut Universitaire de France, Paris, France; \\ ${ }^{6}$ Service d'Urologie, Hôpital Saint-Louis, Paris, France
}

To date four prostate cancer predisposing loci have been mapped: HPC1 (Hereditary Prostate Cancer 1) on 1q24-25, PCaP (Predisposing for Cancer Prostate) on 1q42.2-43, CAPB (Cancer Prostate and Brain) on 1p36, and HPCX on Xq27-28. We examined evidence for linkage to those loci in 64 families from south and west Europe. Genotyping of three (six for PCaP) markers encompassing the candidate regions were performed on 221 individuals including 159 affected patients. The resulting data were analysed using both parametric and non parametric linkage methods. No significant evidence of linkage to HPC1, CAPB, or HPCX was found either in the whole population or when pedigrees were stratified according to criteria specific to each locus. By contrast, results in favour of linkage to PCaP locus were observed with maximum multipoint NPL and HLOD scores of $2.8(P=0.0026)$ and 2.65 respectively. Homogeneity analysis performed with multipoint LOD scores gave an estimated proportion of families with linkage to this locus up to $50 \%$. Particularly, families with an earlier age at diagnosis ( $\leqslant 65$-years-old) contributed significantly to the evidence of linkage with a maximum multipoint NPL score of $2.03(P=0.024)$. Those results suggest that PCaP is the most frequent known locus predisposing to hereditary prostate cancer cases from families from south and west Europe. European Journal of Human Genetics (2001) 9, 135-142.

Keywords: hereditary; prostate cancer; linkage analysis; genetic heterogeneity; PCaP (Predisposing for Cancer Prostate)

\section{Introduction}

Prostate cancer, with 85000 new cases per year, is the most common cancer diagnosed among men over 50-years-old in Europe. This affection annually causes the death of 35000 men in the European Union. Epidemiological studies have revealed a familial clustering of prostate cancer cases which is in favour of an inherited form of the disease. ${ }^{1-3}$ In particular, men with a first-degree relative affected had a higher risk to develop prostate cancer, as compared to those with no affected relatives. This risk tends to increase with increasing

\footnotetext{
${ }^{*}$ Correspondence: Dr Géraldine Cancel-Tassin, CeRePP, genopole ${ }^{\mathbb{B}}$, 4 rue Pierre Fontaine, 91000 EVRY, France. Tel: +33 1608789 70; Fax: +331608789 89; E-mail: g.cancel@cerepp.org Received 24 May 2000; revised 22 September 2000; accepted 29 September 2000
}

numbers of affected family members. ${ }^{3}$ Segregation analysis of families has indicated that hereditary prostate cancer may be due to a rare, highly penetrant, autosomal dominant gene(s). ${ }^{2,4,5}$

In order to identify this gene, several genome-wide screens were performed on familial prostate cancer cases. These have led to the mapping of four predisposing genes. The first localised was HPC1 (Hereditary Prostate Cancer 1) on 1q24$25{ }^{6}$ This locus was identified in a study including North American and Swedish families with at least three affected individuals. Assuming heterogeneity, a maximum multipoint LOD score of 5.43 was found, with an estimated $34 \%$ of the families being linked. Extension of this analysis to a larger number of families from the same origin showed that a subset of pedigrees with an earlier age at diagnosis and at least five prostate cases was mostly responsible for the evidence of 
linkage. ${ }^{7}$ In order to confirm this localisation, several linkage analyses were performed with controversial results. Some studies concluded absence of linkage within the selected population, ${ }^{8-11}$ whereas others show weak confirmation. ${ }^{12,13}$ The strongest confirmation for this localisation was obtained by the examination of Utah families, ${ }^{14}$ with a maximum three-point LOD score of 2.43. Again, in this study, the younger age at diagnosis pedigrees provided stronger evidence of linkage to HPC1. Recently, a large linkage analysis performed by the International Consortium on Prostate Cancer Genetic (ICPCG) on 772 families from various origins has indicated that the estimated proportion of families linked to this locus was closer to $6 \%$ rather than $30 \%$. $^{15}$

The second locus called PCaP (Predisposing for Cancer Prostate) was identified on 1q42.2-43 by a combined analysis of French and German families. ${ }^{10}$ This result was not confirmed by other groups ${ }^{16}$ but positive LOD scores suggestive of linkage were obtained when families were stratified. Indeed, evidence of linkage was observed among a small subset of families with $\geqslant 5$ affected individuals ${ }^{17}$ or with pedigrees which meet the same criterion, had an average age at diagnosis $<66$ years and male to male transmission. ${ }^{18}$

Examination of 360 families collected in North America, Finland and Sweden led to the identification of a third locus, termed HPCX, on Xq27-28. ${ }^{19}$ In the overall population, the proportion of linked pedigrees was close to $16 \%$ but reached $41 \%$ in the Finnish subgroup. The results supporting this localisation were further obtained in another analysis of 153 American families. ${ }^{20}$ In this report, the most significant evidence of linkage to this locus was found in pedigrees without male to male transmission and with early-onset prostate cancer.

The last gene which has been localised on 1p36 seemed to be predisposing for brain and prostate cancer. Indeed, strong evidence of linkage to this locus, CAPB, was obtained with 12 families showing both hereditary prostate cancer and a history of brain tumours. ${ }^{21}$ By contrast, results that do not support linkage to this locus were presented by an independent analysis of 13 pedigrees presenting the same clinical profile. $^{18}$

Considering the genetic heterogeneity and the controversial results observed within the different populations, we have selected 64 families from south and west Europe and performed a linkage analysis with markers from the four candidate regions, in order to estimate the frequency of each locus in this subset of pedigrees.

\section{Materials and methods \\ Families}

Sixty-four families with at least three affected individuals were selected for linkage analysis. This subset of families included the 37 French pedigrees that were analysed in our previous genome-wide screen. ${ }^{10}$ They were all from south and west Europe: 61 were from France, two from Spain and one from Italy. While we deliberately excluded families with northern European and African origins, some families from France appeared to be originating from Spain, Portugal and Italy. The average number of affected individuals per pedigree was 3.75 (range $3-7$ ). The number of genotyped individuals was 221 including 159 affected men (mean, 2.5 per family; range $2-5$ ). All affected men who were genotyped had their prostate cancer confirmed by a pathological record. The average age at diagnosis was 66.4 years (range: 48 - 85 years). Twenty-five families had an average age at diagnosis $\leqslant 65$ years-old.

\section{Markers and genotyping}

DNA was extracted from peripheral blood using standard methods. Three markers covering the candidate interval were selected for each locus, except for PCaP, where six markers were analysed: D1S2678, D1S2670, D1S2785, D1S321, D1S304 and D1S2842. For HPC1 locus, D1S2883, D1S158 and D1S413 were studied. DXS984, DXS8106 and DXS1200 were chosen for linkage analysis of the HPCX locus and D1S434, D1S407 and D1S436 for the CAPB locus. PCR amplification was performed with fluorescently labelled primers. In a $15 \mu \mathrm{l}$ final volume, $50 \mathrm{ng}$ of genomic DNA, $250 \mu \mathrm{M}$ dNTP, $1 \mu \mathrm{M}$ each primer, $0.6 \mathrm{U}$ Taq polymerase Appligène, $1.5 \mathrm{~mm} \mathrm{MgCl}_{2}$ were added. Reactions were cycled as follows: $7 \mathrm{~min}$ at $95^{\circ} \mathrm{C}$, then 12 cycles of $15 \mathrm{~s}$ at $94^{\circ} \mathrm{C}, 15 \mathrm{~s}$ at $55^{\circ} \mathrm{C}, 30 \mathrm{~s}$ at $72^{\circ} \mathrm{C}$, then 22 cycles of $15 \mathrm{~s}$ at $89^{\circ} \mathrm{C}, 15 \mathrm{~s}$ at $55^{\circ} \mathrm{C}, 30 \mathrm{~s}$ at $72^{\circ} \mathrm{C}$; followed by a final extension step of $10 \mathrm{~min}$ at $72^{\circ} \mathrm{C}$. PCR products were loaded on a $5 \%$ denaturing polyacrylamide gel and detected by use of an ABI Prism 377 DNA sequencer. Genotypes were determined with Genescan Analysis 3.1 and Genotyper 2.0 softwares (Perkin-Elmer).

\section{Statistical analysis}

Two-point linkage analyses were performed with the package LINKAGE using the FASTLINK 3.0 implementation. ${ }^{22,23}$ Parametric and non parametric multipoint scores were computed by use of GENEHUNTER. ${ }^{24}$ For the parametric analyses, two different genetic models, previously described in Berthon et al. ${ }^{10}$ were used. In brief, they all assumed an autosomal dominant mode of inheritance with a disease allele frequency of 0.003. In these models, four age-dependent penetrance classes were established based on the estimated cumulative risks of prostate cancer for gene carriers in the segregation analysis of Carter et al. ${ }^{3}$ The penetrances for the susceptible genotypes were: 0.01 at age $<40$ years, 0.1 at age $40-55$ years, 0.5 at age $55-70$ years and 0.9 at age $<70$ years. The two models differed by the phenocopy rate. The phenocopy rate was 0.1 , in all age classes in model M1 and 10\% of the susceptible-genotype penetrance, in all age classes, for the second model M2. Linkage in the presence of heterogeneity was assessed by use of Smith's admixture test for hetero- 
geneity (HOMOG program). ${ }^{25}$ Allele frequencies were estimated from the data set.

\section{Results}

We examined evidence for linkage to four prostate cancer predisposing loci in 64 families from south and west Europe. Statistical analyses for each locus were performed with two different models previously described by Berthon et al. ${ }^{10}$ Consistent results were found with the M1 and M2 models, with lower positive or negative values for M2.

\section{HPC1 (1q24-25)}

When considering the 64 families, no evidence of linkage was observed with the three selected markers from the HPC1 candidate interval. Using either model, the three markers each had negative or very small positive two-point LOD scores. All values were under 0.25. Multipoint LOD scores were also negative across the entire interval, and only reached a maximum of -12.25 for model M1 and -1.81 for model M2 at D1S413. Under hypothesis of heterogeneity, maximum multipoint LOD score (HLOD) was 0.07 with $\alpha$ (proportion of families with linkage) $=0.075$ for model M1, and 0.01 with $\alpha=0.109$ for M2. With the non parametric all pair statistical analysis, which is independent of the genetic model, the maximum NPL score was $0.28(P=0.38)$ at D1S2883.

Previous reports showed that evidence for the linkage to HPC1 was provided mostly by families with an earlier age of diagnosis ( $\leqslant 65$ years). ${ }^{7,26}$ We then reanalysed our data in a subset of 25 families meeting this criterion. No more evidence of linkage was found, while two-point and multipoint LOD scores remained negative for the entire interval. Assuming heterogeneity, a maximum multipoint HLOD of 0.107 with $\alpha=0.125$ was found at D1S2883, with the model M1. The maximum non parametric multipoint NPL score was $0.58(P=0.28)$ at the same position.

\section{PCaP (1q42.2-43)}

We have analysed the 64 European families with six markers from the PCaP region. LOD scores from the two-point parametric analysis using both models of inheritance of prostate cancer are given in Table 1 . The highest LOD scores were observed with D1S2785 and D1S2842 markers. A peak LOD score of 2.56 at $\theta=0.1$ was obtained with the model M1 at D1S2842, whereas with the same marker, it was 2.09 at $\theta=0.00$ for the M2 model. With regard to the 27 families who were not included in our previous study, ${ }^{10}$ the highest positive two-point LOD scores were obtained with the D1S2842 marker, with a maximum of 0.79 for the M1 model and 0.86 for the M2 model.

For the all data set of families, multipoint LOD scores considering five markers (D1S2678, D1S2670, D1S2785, D1S321 and D1S2842) were negative across the entire interval with model M1. In contrast, positive LOD scores were obtained under the M2 model with a maximum reaching 2.59 (Figure 1). Under the hypothesis of heterogeneity, models M1 and M2 respectively gave a maximum HLOD of $2.65(\alpha$, proportion of linked families $=0.48)$ and $2.59(\alpha=0.995)$ between D1S321 and D1S2842 markers. Linkage homogeneity was tested using the multipoint data for each model with the HOMOG program. Under M1, the odds ratio was $439 / 1$ in favour of linkage with heterogeneity as opposed to no linkage, with an $\alpha$ being $50 \%$. No heterogeneity was found with M2. Results of the non parametric analysis were also in favour of linkage, with a maximum multipoint NPL score of $2.86(P=0.0026)$ at a position corresponding to D1S2785.

Our previous report on $\mathrm{PCaP}$ localisation demonstrated that families with an earlier age at diagnosis largely contributed to the evidence of linkage. ${ }^{10}$ Statistical analysis was then performed on the twenty-five families with mean age at diagnosis under 66 years of age (Figure 2). With the M1 model, a maximum two-point LOD score of 2.23 at $\theta=0.05$ was observed with the D1S2842 marker. In contrast, the highest LOD score obtained with the M2 model was 1.15 at $\theta=0.00$ with D1S2785. Negative or slightly positive parametric multipoint LOD scores were observed across the entire interval using either model in this subgroup of families. By contrast, when heterogeneity is assumed, HLOD scores were positive along the same interval and using either the M1 or M2 model, reached 1.23 and 0.53 with respectively $58 \%$ and $78 \%$ of linked families at a position corresponding to D1S2842. Again, non parametric multipoint NPL scores were suggestive of linkage to the PCaP locus with a maximum of $2.03(P=0.02)$ at D1S2842.

\section{HPCX (Xq27-28)}

With the model M1, negative two-point parametric LOD scores were observed at the recombination rate of 0.00 for the three markers from the HPCX region (data not shown). All LOD scores at this recombination fraction were under -2.0 which is considered to be significant evidence against linkage. Slightly positive LOD scores were only reached at higher recombination rates: 0.41 for DXS1200, 0.20 for DXS8106 and 0.39 for DXS984 $(\theta=0.3)$. With the model M2, low positive two-point lod scores were observed with the DXS1200 marker (maximum two-point LOD score of 0.35 at $\theta=0.1$ ). Multipoint LOD scores, across the entire interval were also negative with the maximum of -7.04 for model M1 and -0.51 for M2, close to DXS1200. Under the assumption of heterogeneity, the largest LOD score obtained with the M1 model was 0.14 , with $\alpha=0.107$, whereas it was 0.06 with $\alpha=0.272$, for the M2 model, at a position corresponding to DXS1200. The NPL scores were slightly negative across the entire interval, except at DXS1200 position where a low positive NPL value of 0.03 is found $(P=0.48)$.

We stratified the families according to whether or not they had male to male transmission. Eighteen of our pedigrees do 
Table 1 Two-Point LOD scores within the 1q42.2-43 PCaP region, for all the 64 European families, the 27 newly analysed ones and the 25 pedigrees with an earlier age at diagnosis ( $\leqslant 65$-years-old)

\begin{tabular}{|c|c|c|c|c|c|c|c|c|}
\hline \multirow{2}{*}{ Model and marker } & \multirow[b]{2}{*}{ Distance (cM) } & \multicolumn{7}{|c|}{$L O D$ score at $\theta=$} \\
\hline & & 0.0 & 0.01 & 0.05 & 0.1 & 0.2 & 0.3 & 0.4 \\
\hline \multicolumn{9}{|l|}{ All families } \\
\hline \multicolumn{9}{|l|}{ M1: } \\
\hline D1S2678 & & -5.1 & -4.18 & -2.11 & -0.83 & 0.14 & 0.24 & 0.09 \\
\hline D1S2670 & 7.2 & -6.13 & -4.46 & -1.15 & 0.49 & 1.26 & 0.88 & 0.30 \\
\hline D1S2785 & 2.2 & -2.26 & -0.99 & 1.30 & 2.20 & 2.12 & 1.25 & 0.40 \\
\hline D1S321 & 2.3 & -3.03 & -2.38 & -0.97 & -0.17 & 0.36 & 0.31 & 0.11 \\
\hline D1S304 & 0 & -3.39 & -2.66 & -1.09 & -0.23 & 0.29 & 0.25 & 0.09 \\
\hline D1S2842 & 5.3 & -0.31 & 0.56 & 2.07 & 2.56 & 2.18 & 1.25 & 0.39 \\
\hline \multicolumn{9}{|l|}{ M2: } \\
\hline D1S2678 & & 0.30 & 0.33 & 0.41 & 0.44 & 0.35 & 0.18 & 0.05 \\
\hline D1S2670 & 7.2 & 0.30 & 0.37 & 0.56 & 0.64 & 0.53 & 0.27 & 0.06 \\
\hline D1S2785 & 2.2 & 1.79 & 1.78 & 1.68 & 1.47 & 0.97 & 0.47 & 0.12 \\
\hline D1S321 & 2.3 & 0.06 & 0.11 & 0.23 & 0.31 & 0.30 & 0.18 & 0.06 \\
\hline D1S304 & 0 & -0.01 & 0.02 & 0.11 & 0.16 & 0.15 & 0.08 & 0.02 \\
\hline D1S2842 & 5.3 & 2.09 & 2.07 & 1.90 & 1.65 & 1.07 & 0.52 & 0.13 \\
\hline \multicolumn{9}{|c|}{27 newly analysed families } \\
\hline \multicolumn{9}{|c|}{ M1: } \\
\hline D1S2678 & & -2.11 & -1.66 & -0.67 & -0.11 & 0.24 & 0.19 & 0.06 \\
\hline D1S2670 & 7.2 & -4.69 & -3.88 & -2.09 & -1.03 & -0.18 & 0.03 & 0.02 \\
\hline D1S2785 & 2.2 & -3.35 & -2.63 & -1.15 & -0.35 & 0.17 & 0.18 & 0.06 \\
\hline D1S321 & 2.3 & -2.38 & -2.04 & -1.25 & -0.72 & -0.21 & -0.03 & 0.00 \\
\hline D1S304 & 0 & -1.40 & -1.23 & -0.78 & -0.47 & -0.16 & -0.04 & -0.01 \\
\hline D1S2842 & 5.3 & -0.92 & -0.49 & 0.34 & 0.69 & 0.70 & 0.42 & 0.12 \\
\hline \multicolumn{9}{|l|}{ M2: } \\
\hline D1S2678 & & 0.83 & 0.81 & 0.74 & 0.63 & 0.40 & 0.19 & 0.05 \\
\hline D1S2670 & 7.2 & -0.13 & -0.10 & -0.01 & 0.05 & 0.08 & 0.05 & 0.01 \\
\hline D1S2785 & 2.2 & 0.21 & 0.22 & 0.23 & 0.23 & 0.17 & 0.08 & 0.02 \\
\hline D1S321 & 2.3 & -0.08 & -0.07 & -0.01 & 0.04 & 0.07 & 0.05 & 0.02 \\
\hline D1S304 & 0 & -0.15 & -0.13 & -0.09 & -0.05 & -0.02 & -0.00 & 0.00 \\
\hline D1S2842 & 5.3 & 0.86 & 0.85 & 0.79 & 0.69 & 0.45 & 0.23 & 0.06 \\
\hline \multicolumn{9}{|c|}{25 families with age at diagnosis $\leqslant 65$-years-old } \\
\hline M1: & & & & & & & & \\
\hline D1S2678 & & -2.32 & -1.95 & -1.05 & -0.44 & 0.05 & 0.12 & 0.05 \\
\hline D1S2670 & 7.2 & -4.86 & -3.85 & -1.78 & -0.62 & 0.22 & 0.30 & 0.14 \\
\hline D1S2785 & 2.2 & 1.06 & 1.39 & 1.85 & 1.89 & 1.45 & 0.81 & 0.26 \\
\hline D1S321 & 2.3 & -2.22 & -1.89 & -1.12 & -0.62 & -0.16 & -0.01 & 0.01 \\
\hline D1S304 & 0 & -1.38 & -1.10 & -0.49 & -0.14 & 0.09 & 0.09 & 0.03 \\
\hline D1S2842 & 5.3 & 1.99 & 2.14 & 2.23 & 2.05 & 1.45 & 0.80 & 0.26 \\
\hline \multicolumn{9}{|l|}{ M2: } \\
\hline D1S2678 & & -0.53 & -0.48 & -0.31 & -0.16 & -0.01 & 0.02 & 0.01 \\
\hline D1S2670 & 7.2 & -1.10 & -1.00 & -0.68 & -0.41 & -0.13 & -0.04 & -0.02 \\
\hline D1S2785 & 2.2 & 1.15 & 1.13 & 1.04 & 0.90 & 0.58 & 0.28 & 0.06 \\
\hline D1S321 & 2.3 & -0.45 & -0.41 & -0.28 & -0.16 & -0.03 & 0.00 & 0.00 \\
\hline D1S304 & 0 & -0.01 & 0.00 & 0.04 & 0.07 & 0.07 & 0.04 & 0.01 \\
\hline D1S2842 & 5.3 & 1.05 & 1.02 & 0.92 & 0.78 & 0.48 & 0.23 & 0.05 \\
\hline
\end{tabular}

not show this kind of transmission. An analysis of these families did not suggest linkage. At $\theta=0.00$, all two-point LOD scores were negative, except those with DXS1200 and model M2. The maximum values were 0.15 at $\theta=0.3$ with the marker DXS1200 and model M1, and 0.19 at $\theta=0.1$ with the same marker and M2. Multipoint LOD scores with or without heterogeneity were negative across the entire region with either model. Multipoint analysis of this group of families resulted in a maximum NPL score -0.21 at DXS1200 $(P=0.57)$.

\section{CAPB (1p36)}

When considering the 64 European families, no evidence of linkage was observed with the three markers encompassing the candidate region for CAPB. Parametric twopoint and multipoint LOD scores were negative at all 


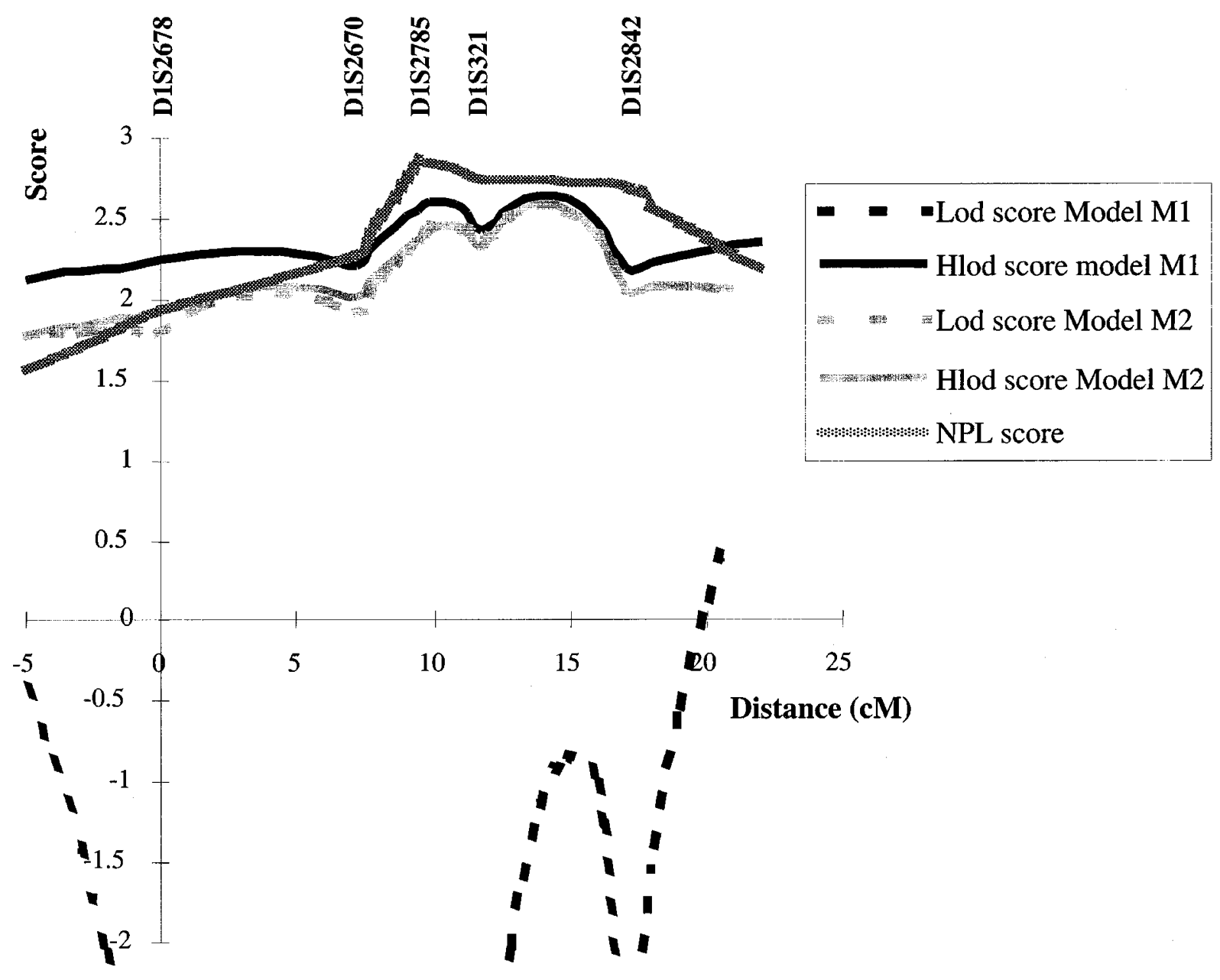

Figure 1 Multipoint parametric LOD scores and non parametric NPL scores for 64 families, computed with GENEHUNTER, on a map of five markers from the PCaP region. HLODs are maximized on $\alpha$.

recombination fractions. Only when heterogeneity is assumed, were slightly positive values obtained. Indeed, there was a maximum multipoint HLOD of 0.057, with the estimated proportion of linked families, $\alpha$, being $3.3 \%$ with model M1; and of 0.147 with an $\alpha$ of $2.8 \%$ for model M2. Model-independent NPL scores were negative across the entire interval, with a maximum NPL score of -0.08 $(P=0.53)$ at D1S436.

The CAPB locus seems to be most strongly associated to families showing both a familial prostate cancer and a history of brain cancer. $^{21}$ Only six of our pedigrees included one case of brain cancer. Linkage to this locus was reassessed for those families. Results were not in favour of linkage with negative parametric two-point and multipoint LOD scores. Even if heterogeneity was assumed, multipoint HLOD scores remained negative across the entire interval. Non parametric multipoint NPL scores were also negative, with a maximum NPL score of -0.80 $(P=0.78)$ at D1S436.

\section{Discussion}

In this study, we have examined linkage analysis to the four known prostate cancer predisposing loci in a selected population. This subset of families were from south and west Europe, mostly from France. In this whole population, no significant evidence of linkage to the HPC1 locus was found. This result agrees well with the data obtained in the genomewide screen that we performed on 37 French and 10 German families. In this previous report, negative two-point LOD scores were observed with each marker selected in the HPC1 region. ${ }^{10}$ Those findings could be explained by the difference in origin of pedigrees. Indeed, the HPC1 locus was identified by linkage analysis of North American and Swedish families. ${ }^{6}$ Suggestive evidence of linkage was then only obtained with North American pedigrees. ${ }^{12}$ In addition, the only study which reached significant statistical results for confirmation of this localisation was obtained by linkage analysis performed on Utah families. ${ }^{14}$ Regarding our results and the finding that positive LOD scores were observed in Utah 


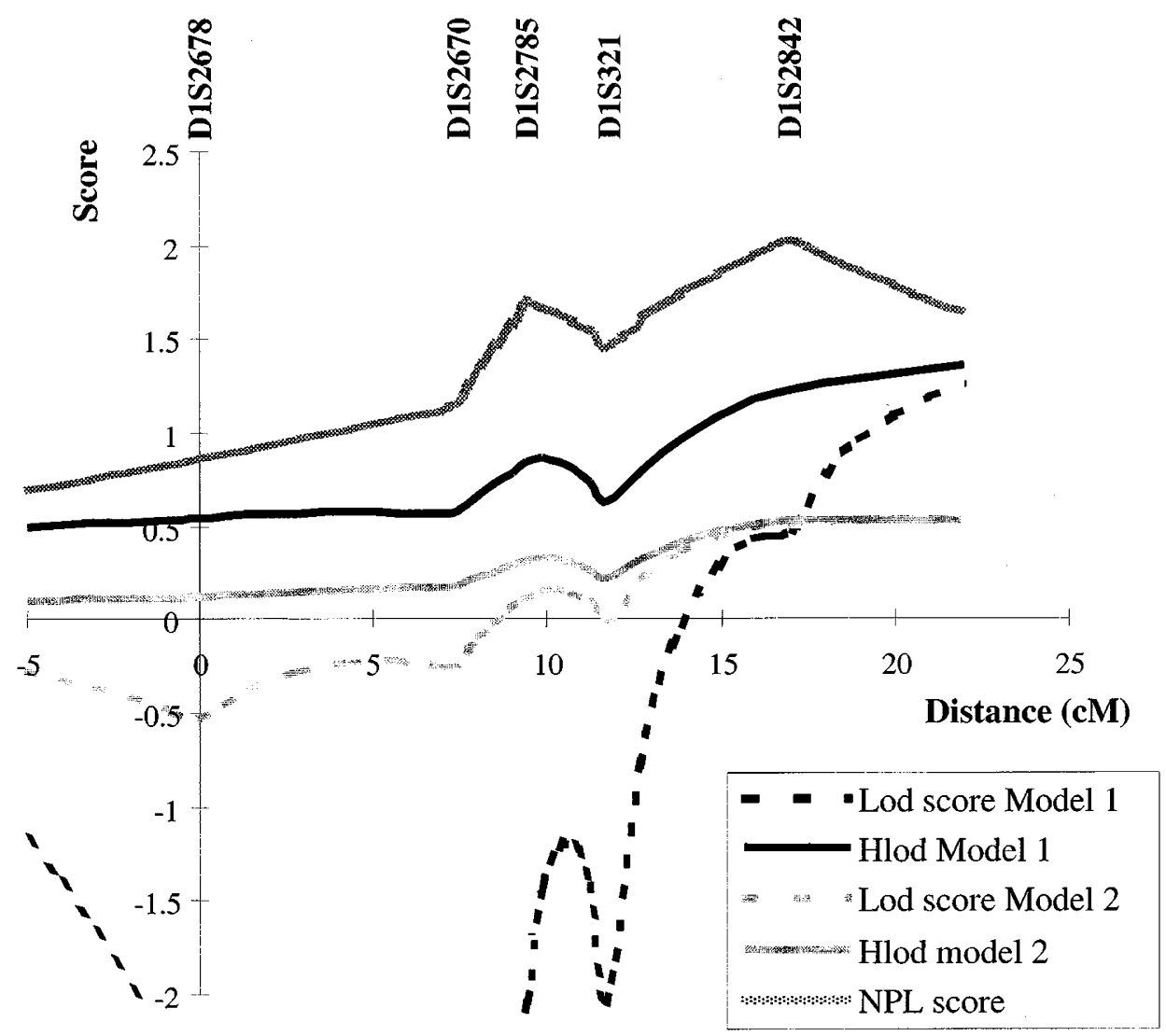

Figure 2 Multipoint parametric LOD scores and non parametric NPL scores for 25 families with an earlier age at onset ( $\leqslant 65$-years-old), computed with GENEHUNTER, on a map of five markers from the PCaP region. HLODs are maximized on $\alpha$.

and Swedish pedigrees, ${ }^{7,14,22}$ we could speculate that this locus is largely implicated in families from northern European origin. Another explanation could be the low frequency of this mutation in hereditary prostate cancer. Indeed, in the first analysis leading to the identification of HPC1, the estimated fraction of linked families reached 34\%, but recently, this locus was reassessed in a vast analysis including 772 northern American and European families. ${ }^{15}$ In this report, the proportion of linked families was $6 \%$. This region may be implicated for a small subset of our pedigrees, but our sample may not be large enough to provide strong evidence of linkage. Indeed, when heterogeneity is assumed, we found low positive LOD scores with a proportion (seven or $11 \%$ depending upon the model used) of our families who might be linked to this locus.

Several studies have indicated that families with an earlier age at diagnosis contributed mostly to the evidence of linkage to HPC1..$^{7,15,22}$ We have reanalysed our data for the 25 families with mean age at diagnosis $\leqslant 65$ years. Even in this subgroup of families, no significant evidence of linkage was observed. The most recent report on HPC1 suggested that this locus might be particular to families with an earlier age at diagnosis, more than four affected individuals and male to male transmission. Only five of our families met those three criteria. This might also explain the absence of linkage that we observed.

Further, we find no significant evidence of linkage to the HPCX locus in our whole population data set. Again the difference observed between our results and those obtained for the HPCX localisation may be due to the origin of the studied populations. Discrepancy in the proportion of linked families was already observed, with $15 \%$ for North American and $40 \%$ for Finnish pedigrees. ${ }^{19}$ If heterogeneity is assumed, only a small fraction of our families (between 11 and $27 \%$ depending on M1 or M2 model) might be linked to this locus. In contrast to the results previously described, ${ }^{19}$ we didn't find evidence of linkage when we stratified our population regarding male to male transmission. Analysis of the 18 families without this kind of transmission gave the strongest results confirming the absence of linkage.

The analysis of the CAPB locus shows no evidence of linkage either when we analysed the whole population or families with a history of brain cancer. The absence of linkage observed in the whole group of pedigrees wasn't surprising 
since this locus was described to be specific of families with brain and prostate cancers. ${ }^{21}$ Considering the seven families showing a history of cerebral tumour, results were again in favour of the absence of linkage to this locus. Similar results were obtained by an independent study including 13 families with prostate and brain cancer. ${ }^{18}$ Those findings suggest that this locus is not the only one responsible for brain and prostate cancer.

In contrast to the results obtained for the other loci, data suggestive of linkage were found with markers from the PCaP candidate region. This analysis of 64 European families, mostly French, extends our previous report corresponding to a genome-wide screen including 37 French and 10 German pedigrees, ${ }^{10}$ and agrees with our data indicating the existence of a prostate cancer predisposing gene on 1q42.2-43. To date, several studies based on Caucasian populations, particularly American, have tried to replicate our results without success. ${ }^{16,17}$ Only a small subset of their families might be linked to PCaP. ${ }^{17,18}$ This suggests that this locus is most specific to families from south and west Europe. As in our previous report, ${ }^{10}$ homogeneity analysis indicated that up to $50 \%$ of the studied families are linked to this locus. Again, we do think that it is probably an overestimation due in part to the number of families with only two fully informative meioses. To estimate better the proportion of linked families, a world-wide analysis encompassing a large number of pedigrees of different origin, similar to those performed for HPC1, is currently being carried out through the ICPCG. This will also indicate the frequency of families showing linkage to this locus regardless of the origins of the pedigrees.

In our previous report, we showed that families with an earlier age at onset contributed strongly to the evidence of linkage. ${ }^{10}$ In this study, evidence of linkage was obtained with families with an age at diagnosis $\leqslant 65$-years-old.

In conclusion, our study contributes to the evidence of a growing genetic heterogeneity among hereditary prostate cancers. Even, if $\mathrm{PCaP}$ is implicated in up to $50 \%$ of the families and either HPC1 or HPCX in a small fraction, a place for other predisposing genes remains. This genetic heterogeneity could in part be explained by the difference of family origins. Indeed, whereas evidence of linkage to HPC1 and HPCX seemed to be most important among families with northern European origin, linkage to $\mathrm{PCaP}$ is most frequent in pedigrees from south and west Europe.

\section{Acknowledgements}

We would like to thank Emmanuelle Guillaume, Marc Guérard, Patrice Morant and Isabelle Gauthier for technical assistance. We acknowledge Dr David J Grausz for critical reading of the manuscript. We would like to thank the families for their participation and the French Association of Urology (AFU) for its continuous support of this programme. We are grateful to the clinicians who referred the families. This work was supported by grants from Association pour la Recherche sur le Cancer (ARC 5441), INSERM (4R004C) and ARITT (IdF 99).

\section{References}

1 Steinberg GD, Carter BS, Beaty TH, Childs B, Walsh PC: Family history and the risk of prostate cancer. Prostate 1990; 17: 337 347.

2 Carter BS, Beaty TH, Steinberg GD, Childs B, Walsh PC: Mendelian inheritance of familial prostate cancer. Proc Natl Acad Sci USA 1992; 89: 3367 - 3371.

3 Carter BS, Bova GS, Beaty TH et al: Hereditary prostate cancer: epidemiologic and clinical features. J Urol 1993; 150: 797 -802.

4 Gronberg H, Damber L, Damber JE, Iselius L: Segregation analysis of prostate cancer in Sweden: support for dominant inheritance. Am J Epidemiol 1997; 146: 552-557.

5 Schaid DJ, McDonnell SK, Blute ML, Thibodeau SN: Evidence for autosomal dominant inheritance of prostate cancer. Am J Hum Genet 1998; 62: $1425-1438$.

6 Smith JR, Freije D, Carpten JD et al: Major susceptibility locus for prostate cancer on chromosome 1 suggested by a genome-wide search. Science 1996; 274: $1371-1374$.

7 Gronberg $\mathrm{H}, \mathrm{Xu}$ J, Smith JR et al: Early age at diagnosis in families providing evidence of linkage to the hereditary prostate cancer locus (HPC1) on chromosome 1. Cancer Res 1997; 57: 4707 - 4709

8 McIndoe RA, Stanford JL, Gibbs M et al: Linkage analysis of 49 high-risk families does not support a common familial prostate cancer-susceptibility gene at 1q24-25. Am J Hum Genet 1997; 61: 347-353.

9 Eeles RA, Durocher F, Edwards S et al: Linkage analysis of chromosome 1q markers in 136 prostate cancer families. Am J Hum Genet 1998; 62: 653-658.

10 Berthon P, Valeri A, Cohen-Akenine A et al: Predisposing gene for early-onset prostate cancer, localized on chromosome 1q42.2-43. Am J Hum Genet 1998; 62: 1416-1424.

11 Goode EL, Stanford JL, Chakrabarti L et al: Linkage analysis of 150 high-risk prostate cancer families at 1q24-25. Genet Epidemiol 2000; 18: $251-275$.

12 Cooney KA, McCarthy JD, Lange E et al: Prostate cancer susceptibility locus on chromosome 1q: a confirmatory study. J Natl Cancer Inst 1997; 89: 955 - 959.

13 Hsieh CL, Oakley-Girvan I, Gallagher RP et al: Prostate cancer susceptibility locus on chromosome 1q: a confirmatory study. $J$ Natl Cancer Inst 1997; 89: 1893 - 1894.

14 Neuhausen SL, Farnham JM, Kort E, Tavtigian SV, Skolnick MH, Cannon-Albright LA: Prostate cancer susceptibility locus HPC1 in Utah high-risk pedigrees. Hum Mol Genet 1999; 8: 2437 2442 .

$15 \mathrm{Xu} \mathrm{J}$ and the International Consortium for Prostate Cancer Genetics: Combined analysis of hereditary prostate cancer linkage to 1q24-25: results from 772 hereditary prostate cancer families from the international consortium for prostate cancer genetics. Am J Hum Genet 2000; 66: 945-957.

16 Whittemore AS, Lin IG, Oakley-Girvan I et al: No evidence of linkage for chromosome 1q42.2-43 in prostate Cancer. Am J Hum Genet 1999; 65: 254-256.

17 Gibbs M, Chakrabarti L, Stanford JL et al: Analysis of chromosome 1q42.2-43 in 152 families with high risk of prostate cancer. Am J Hum Genet 1999; 64: 1087 - 1095.

18 Berry R, Schaid DJ, Smith JR et al: Linkage analyses at the chromosome 1 loci 1q24-25 (HPC1), 1q42.2-43 (PCAP), and 1 p36 (CAPB) in families with hereditary prostate cancer. Am J Hum Genet 2000; 66: 539-546.

$19 \mathrm{Xu} \mathrm{J}$, Meyers D, Freije D et al: Evidence for a prostate cancer susceptibility locus on the X chromosome. Nat Genet 1998; 20: $175-179$.

20 Lange EM, Chen H, Brierley K et al: Linkage analysis of 153 prostate cancer families over a $30-\mathrm{cM}$ region containing the putative susceptibility locus HPCX. Clin Cancer Res 1999; 5: $4013-4020$.

21 Gibbs M, Stanford JL, McIndoe RA et al: Evidence for a rare prostate cancer-susceptibility locus at chromosome 1p36. Am J Hum Genet 1999; 64: 776-787. 
22 Lathrop GM, Lalouel JM, Julier C, Ott J: Strategies for multilocus linkage analysis in humans. Proc Natl Acad Sci USA 1984; 81: 3443-3446.

23 Cottingham Jr RW, Idury RM, Schaffer AA: Faster sequential genetic linkage computations. Am J Hum Genet 1993; 53: 252263.

24 Kruglyak L, Daly MJ, Reeve-Daly MP, Lander ES: Parametric and nonparametric linkage analysis: a unified multipoint approach. Am J Hum Genet 1996; 58: $1347-1363$.
25 Ott J: Analysis of Human Genetic Linkage (revised edition). Johns Hopkins University Press, Baltimore, 1991.

26 Gronberg H, Smith J, Emanuelsson M et al: In Swedish families with hereditary prostate cancer, linkage to the HPC1 locus on chromosome 1q24-25 is restricted to families with early-onset prostate cancer. Am J Hum Genet 1999; 65: 134-140. 